|| ISSN(online): 2589-8698 || ISSN(print): 2589-868X ||

International Journal of Medical and Biomedical Studies Available Online at www.ijmbs.info

NLM (National Library of Medicine ID: 101738825)

Index Copernicus Value 2020: 79.44

\title{
DETECTION OF PNEUMONIA IN PATIENTS USING CHEST X-RAY IMAGES BASED ON CONVOLUTIONAL NEURAL NETWORKS
}

\author{
Abhay Chopde', Farhan Aga², Ananya Kumar ${ }^{3}$ \\ $1,2,3$ Vishwakarma Institute of Technology, Pune 411037, Maharashtra, India
}

Article Info: Received 03 January 22; Accepted 09 February 2022

DOI: https://doi.org/10.32553/ijmbs.v6i2.2406

Corresponding author: Ananya Kumar

Conflict of interest: No conflict of interest.

\begin{abstract}
Pneumonia is a respiratory disease which inflames one or both lungs, and lungs may fill up with fluid. This can cause fever, chills, cough with phlegm or pus. It is a harmful disease that can be fatal in some cases. It is critical to detect pneumonia early, with a model which could help doctors reduce workload and bring in more accuracy in detection of pneumonia.

In this work, we propose a model which is designed for the diagnosis of pneumonia that is trained on digital chest X-ray images to accurately diagnose pneumonic lungs. In the author's opinion, this could benefit the medical field in the near future.
\end{abstract}

Keywords: $C N N$, Keras, Pneumonia

\section{Introduction}

Pneumonia is a common but dangerous respiratory infection of the lungs, which can be fatal in some cases. Common symptoms are difficulty in breathing, fever, chills, cough with pus or phlegm. This condition is more likely to impact people with a weakened immune system, elderly individuals, and children under the age of five. Pneumonia is responsible for the deaths of over three lakh children in India, with children accounting for half of all pneumonia deaths. The majority of the time, the sickness goes unnoticed. If it is diagnosed early enough, many lives can be saved.

Pneumonia is diagnosed using radiological methods like chest X-rays, CT scans of the lungs, MRIs of the chest, ultrasounds of the chest, chest radiographs, and lung biopsy. Even in rare cases, radiologists incorrectly classify viral pneumonia or bacterial imaging, resulting in ineffective treatment and a worsening of the patient's condition. A technique to aid radiologists in identifying pneumonia from chest $\mathrm{X}$-ray pictures is required.

Many Artificial Intelligence (AI) based solutions are currently being applied to handle biomedical issues (breast cancer screening, brain tumour detection, and so on). Because they are affordable and there is plenty of data for feeding different Machine Learning models, Machine Learning and Deep Learning approaches are commonly used on chest X-rays. These models predict disease with accuracy comparable to, if not greater than, that of a single radiologist. CNN's perform exceptionally well when working with photos, and as a result, they are widely recognised among researchers. Transfer learning models with pre-trained weights, such as VGG16, VGG19, and ResNet50, are a few of the most effective Image Net dataset models. We implemented these models and trained them to categorise Xray pictures of the chest into normal or pneumonia detection in a short amount of time, allowing for a faster and earlier diagnosis of pneumonia.

\section{Literature Review}

\section{- CNN or ConvNet}

The Convolutional Neural Network consists of a model which first learns about the features from the images and then assists in classification based on those characteristics.

CNN's fame stems from its excellent classification implementation. CNN was created on the premise of being able to recognise handwritten zip codes. It's a type of Neural network that is feed-forward. Convolutional neural networks are made up of many layers of artificial neurons. The Convolutional Layer is in charge of learning the features of the images input, followed by the Max-Pooling Layer, which performs max-pooling operations by selecting the maximum value from the kernel matrix, and finally, the Fully Connected Layer. To prepare the network for classification skills, all of the neurons in one layer are connected to the neurons in the next layer. The $\mathrm{CNN}$ architecture is very effectively explained in Figure 1. 


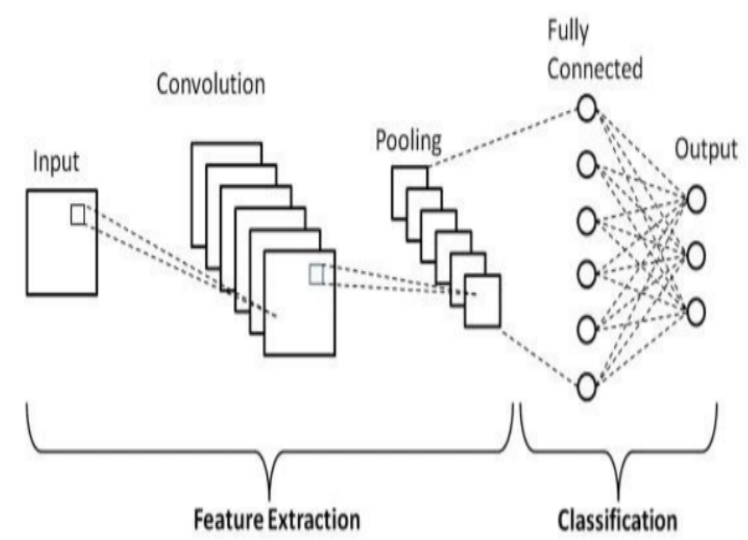

Figure 1: Architecture of the CNN Model

\section{- Pre-trained Transfer Learning}

In this method a machine learning model which is already trained and contains training knowledge from previous training is used. This will make it easier to tackle new problems and help in solving them with a different approach. The work proposes an approach with different points for classification, the first one being Pre-trained, rather than retraining using randomly initialised weights. Very large datasets are used to train these models, but the work consists of a smaller dataset. Through the process of Data Augmentation these models can be implemented on smaller datasets. The many components of transfer learning, as well as its operating model, are depicted in Figure 2.

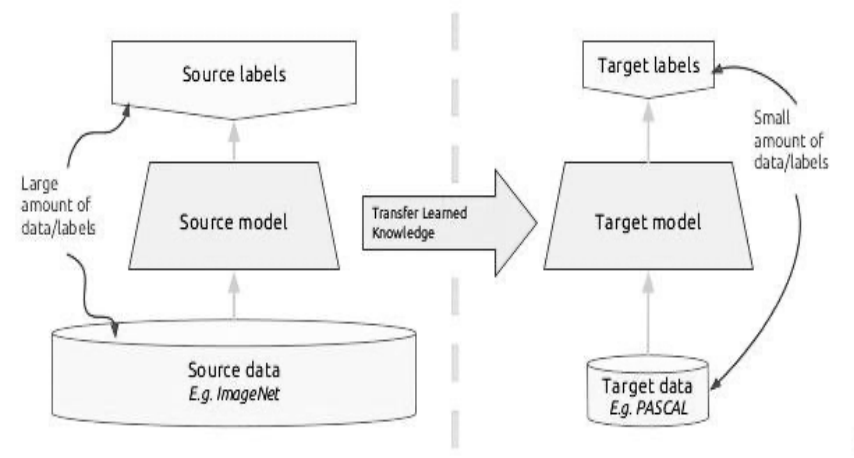

Figure 2: Architecture of the components of Transfer Learning

\section{- Pre-trained CNN}

The work was implemented using a pre-trained Convolutional Neural Network (CNN) Its is known as VGG and it is a different type of CNN model. It was observed that VGG achieved a 92.7 percent accuracy rating. Over fourteen million photos were used to train and test it, and there were thousands of different classes. This is a simple network where $3 \times 3$ convolutional layers are layered on top of each other[11]. The size is lowered with the use of max-pooling. After that, there are two completely connected layers, each with 4,094 nodes, and a SoftMax [8]. The performance was observed using VGG16 and VGG19.

1. A deep ConvNet consisting of 16 layers known as VGG16. 2. A deep ConvNet consisting of 19 layers known as VGG19.
After going through the given methods and learning about them we decided to implement our project using Transfer Learning and VGG16 architecture.

\section{Methodology}

Kaggle.com was used to obtain the data. Our network was fed by X-ray images of the chest. The data consists of a total of five thousand six hundred and eighty digital chest X-ray images and divided into two main categories:

- Category 1: Normal

- Category 2: Pneumonia

A total of 5,206 photos were used to train the model, 634 images were used for testing, and the remaining images were used for the validation dataset. 
Table 1: Representation of Dataset

\begin{tabular}{|l|l|l|}
\hline \multicolumn{1}{|c|}{ Data } & \multicolumn{1}{c|}{ Category } & \multicolumn{1}{c|}{ Number of Images } \\
\hline $\begin{array}{l}\text { Training } \\
\text { Set }\end{array}$ & Normal & 1341 \\
\hline & Pneumonia & 3875 \\
\hline Test Set & Normal & 234 \\
\hline & Pneumonia & 390 \\
\hline $\begin{array}{l}\text { Validation } \\
\text { Set }\end{array}$ & Normal & 8 \\
\hline & Pneumonia & 8 \\
\hline
\end{tabular}

- Samples Of the 2 Categories:

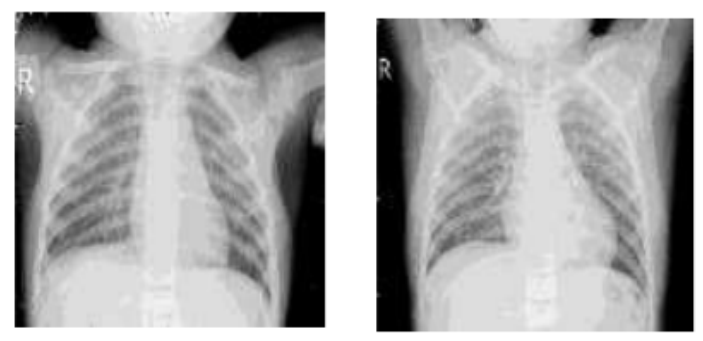

Example of Normal Case

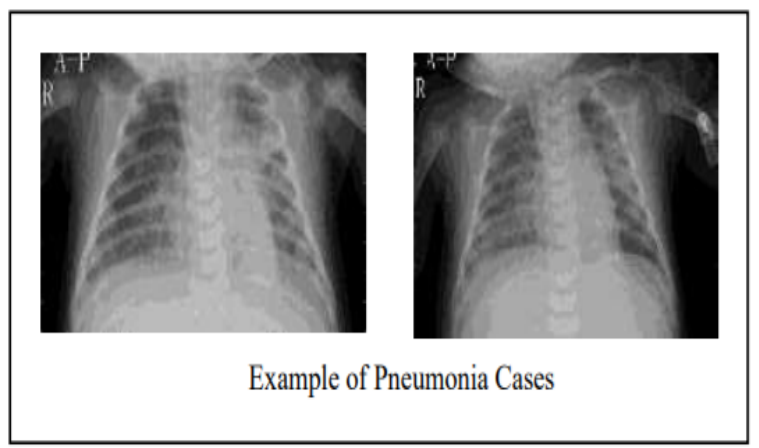

Jupyter Notebook was used in the study to train the model, as well as to evaluate and test the methods. First, we preprocess all of the images, then we do data augmentation on all of the training images, and last, we train using the VGG16 algorithm which we test on our dataset.

Table 2: Parameter used for training

\begin{tabular}{|l|l|l|l|}
\hline Software & Pretrained CNN & Image Size & Optimizer Models \\
\hline Jupyter Notebook & VGG16 & $224 \times 224$ & Adam \\
\hline
\end{tabular}




\section{- Pre-processing}

While developing a machine learning model the first and the foremost step which is very important is Data Preprocessing, similarly another critical requirement is that the input images should be resized to the same size i.e. $224 \times 224$ and passed through the three techniques. The image was resized to $224 \times 224$ for the algorithm, VGG16. All of the photos were also normalised to the appropriate architecture.

\section{- Data Augmentation}

We seek the best solution for X-ray pneumonia diagnosis, and we know that CNN's perform better with huge datasets. We understand that our database is currently somewhat little.
Because we have a smaller sample size, the parameters are harmed, and the generalisation is weak. We typically don't have large datasets to train these algorithms, therefore we use a technique called data augmentation to extend the available dataset. This method is useful since it overcomes the limitation of a small dataset, and the algorithms that are used can be revised thoroughly, so that the model can help to predict pneumonia in an efficient manner. One of the benefits of employing this strategy is that it reduces data overfitting by increasing the size of the train data. As a result, we don't have to be concerned about overfitting.

The following settings are utilised in image augmentation:

Table 3: Augmentation Settings

\begin{tabular}{|l|c|}
\hline \multicolumn{1}{|c|}{ Technique } & Setting \\
\hline rescale & $1 . / 255$ \\
\hline shear_range & 0.2 \\
\hline zoom_range & 0.2 \\
\hline horizontal_flip & True \\
\hline
\end{tabular}

First, the data were preprocessed, which includes cleaning the raw data and removing noise, before the data was ready for training. The data was then processed more effectively, using data augmentation techniques, which increases the data by adding modified copies of the existing data. Because the weights are already fixed, the layers containing the algorithms and techniques utilised in the work were excluded from the training process. The last layer of the VGG16 model was set as False due to the fact that the VGG16 can categorise many categories at a time, but the work requires it to categorise just two. These steps were performed to lessen the computational load, similarly 2 more layers known as the Flatten and the Dense layers were added using an activation function which is known as the softmax activation function. Adam optimizer was the optimizer that was used.

The summary of the model can be observed in the figure given below.

\begin{tabular}{|c|c|c|}
\hline Layer (type) & Output Shape & Param \# \\
\hline input_3 (InputLayer) & {$[($ None, 224, 224, 3) $]$} & 0 \\
\hline block1_conv1 (Conv2D) & (None, 224, 224, 64) & 1792 \\
\hline block1_conv2 (Conv2D) & (None, 224, 224, 64) & 36928 \\
\hline block1_pool (MaxPooling2D) & (None, $112,112,64$ ) & 0 \\
\hline block2_conv1 (Conv2D) & (None, $112,112,128$ ) & 73856 \\
\hline block2_conv2 (Conv2D) & (None, $112,112,128$ ) & 147584 \\
\hline block2_pool (MaxPooling2D) & (None, $56,56,128$ ) & 0 \\
\hline block3_conv1 (Conv2D) & (None, 56, 56, 256) & 295168 \\
\hline block3_conv2 (Conv2D) & (None, 56, 56, 256) & 590080 \\
\hline block3_conv3 (Conv2D) & (None, 56, 56, 256) & 590080 \\
\hline block3_pool (MaxPooling2D) & (None, 28, 28, 256) & 0 \\
\hline block4_conv1 (Conv2D) & (None, 28, 28, 512) & 1180160 \\
\hline
\end{tabular}




\begin{tabular}{|c|c|c|}
\hline block4_conv2 (Conv2D) & (None, 28, 28, 512) & 2359808 \\
\hline block4_conv3 (Conv2D) & (None, 28, 28, 512) & 2359808 \\
\hline block4_pool (MaxPooling2D) & (None, 14, 14, 512) & 0 \\
\hline block5_conv1 (Conv2D) & (None, 14, 14, 512) & 2359808 \\
\hline block5_conv2 (Conv2D) & (None, 14, 14, 512) & 2359808 \\
\hline block5_conv3 (Conv2D) & (None, 14, 14, 512) & 2359808 \\
\hline block5_pool (MaxPooling2D) & (None, 7, 7, 512) & 0 \\
\hline flatten_2 (Flatten) & (None, 25088) & 0 \\
\hline dense_2 (Dense) & (None, 2) & 50178 \\
\hline \multicolumn{3}{|c|}{ 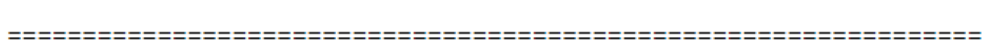 } \\
\hline \multicolumn{3}{|c|}{$\begin{array}{l}\text { Total params: } 14,764,866 \\
\text { Trainable params: } 50,178 \\
\text { Non-trainable params: } 14,714,688\end{array}$} \\
\hline
\end{tabular}

\section{Figure 3: Summary Of Model}

\section{RESULTS AND DISCUSSIONS}

We compared the performance of the ConvNet model VGG16. We achieved a 96.9 per cent accuracy for VGG16. Validation accuracy was 90.8 per cent when tested on validation data.. Accuracy and loss graphs have also been created and are presented in Figure 5

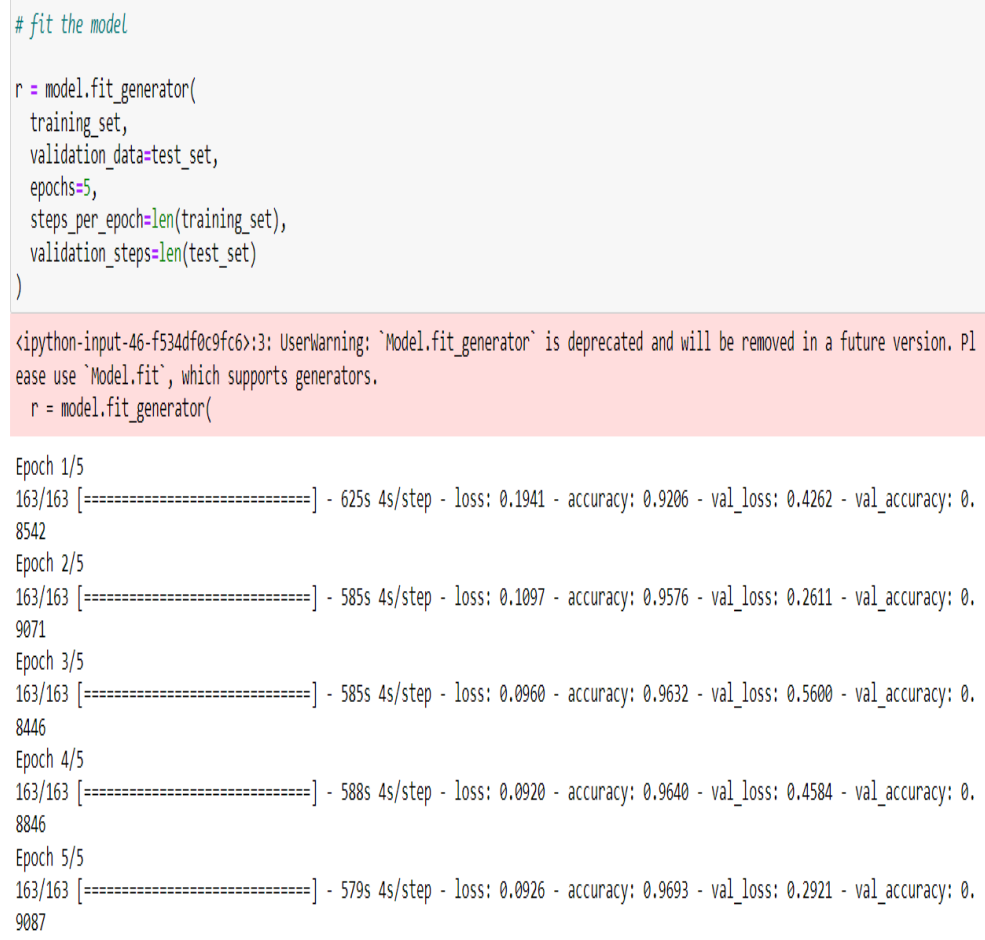

Fig 4: Completion of model training 
The plot for the accuracy and loss of the model is as shown below:
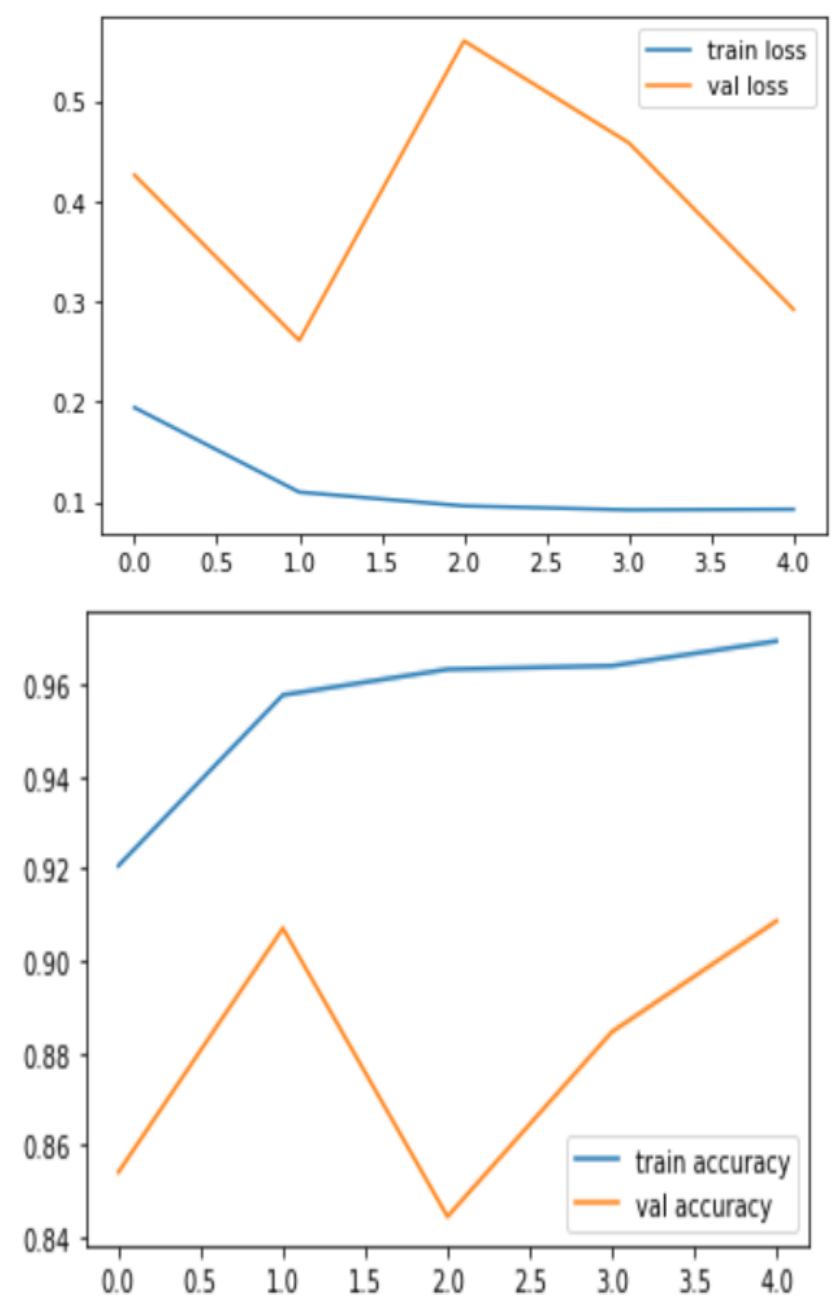

\section{Conclusion}

The model presents transfer learning with implementing CNN, specifically VGG16, for the diagnosis of pneumonia in a very efficient manner. $\mathrm{CNN}$, which stands for Convolutional Neural Network, is a deep learning algorithm, which takes an image, assigns importance to various objects or aspects of the image. Its main attributes could be that it consists of convolution layers, pooling layers, activation layers etc. VGG is a specific convolutional neural network designed for classification and localization. For CNN, the accuracy we got was $96 \%$. Pneumonia is a common lung infection that affects one or both lungs. Pneumonia is a lethal disease and is a reason for mortality. This disease is a cause of a lot of deaths among children. There is always a solution for this, which is timely intervention and treatment of this disease. Precision and Recall models for VGG16 show a great performance for the diagnosis of this infection, which implies great accuracy. Hence, it can be a great contribution.

\section{References}

1. S. Shah, H. Mehta and P. Sonawane, "Pneumonia Detection Using Convolutional Neural Networks," 2020 Third International Conference on Smart Systems and Inventive Technology (ICSSIT), 2020, pp. 933-939, DOI: 10.1109/ICSSIT48917.2020.9214289.

2. D. Varshni, K. Thakral, L. Agarwal, R. Nijhawan and A. Mittal, "Pneumonia Detection Using CNN based Feature Extraction," 2019 IEEE International Conference on Electrical, Computer and Communication Technologies (ICECCT), 2019, pp. 17, DOI: 10.1109/ICECCT.2019.8869364.

3. A. Tilve, S. Nayak, S. Vernekar, D. Turi, P. R. Shetgaonkar and S. Aswale, "Pneumonia Detection Using Deep Learning Approaches," 2020 International 
Conference on Emerging Trends in Information Technology and Engineering (ic-ETITE), 2020, pp. 1-8, DOI: 10.1109/ic-ETITE47903.2020.152.

4. J. Garstka and M. Strzelecki, "Pneumonia detection in X-ray chest images based on convolutional neural networks and data augmentation methods," 2020 Signal Processing: Algorithms, Architectures, Arrangements, and Applications (SPA), 2020, pp. 18-23, DOI: 10.23919/SPA50552.2020.9241305.

5. J. Zhang et al., "Viral Pneumonia Screening on Chest XRays Using Confidence-Aware Anomaly Detection," in IEEE Transactions on Medical Imaging, vol. 40, no. 3, pp. 879-890, March 2021, DOI: 10.1109/TMI.2020.3040950.

6. Z. -Y. Yang and Q. Zhao, "A Multiple Deep Learner Approach for X-Ray Image-Based Pneumonia Detection," 2020 International Conference on Machine Learning and Cybernetics (ICMLC), 2020, pp. 70-75, DOI: 10.1109/ICMLC51923.2020.9469043.

7. K. S. and D. Radha, "Analysis of COVID-19 and Pneumonia Detection in Chest X-Ray Images using Deep Learning," 2021 International Conference on Communication, Control and Information Sciences (ICCISc), 2021, pp. 1-6, DOI: 10.1109/ICCISc52257.2021.9484888.
8. M. Talo, "Pneumonia Detection from Radiography Images using Convolutional Neural Networks," 2019 27th Signal Processing and Communications Applications Conference (SIU), 2019, pp. 1-4, DOI: 10.1109/SIU.2019.8806614.

9. X. Wei, Y. Chen and Z. Zhang, "Comparative Experiment of Convolutional Neural Network (CNN) Models Based on Pneumonia X-ray Images Detection," 2020 2nd International Conference on Machine Learning, Big Data and Business Intelligence (MLBDBI), 2020, pp. 449-454, DOI: 10.1109/MLBDBI51377.2020.00095.

10. T. H. Pingale and H. T. Patil, "Analysis of Cough Sound for Pneumonia Detection Using Wavelet Transform and Statistical Parameters," 2017 International Conference on Computing, Communication, Control and Automation (ICCUBEA), 2017, pp. 1-6, DOI: 10.1109/ICCUBEA.2017.8463900.

11. Maahi Patel, Ayush Sojitra, Zeel Patel, Mohammed Husain Bohara, 2021, Pneumonia Detection Using Transfer Learning, INTERNATIONAL JOURNAL OF ENGINEERING RESEARCH \& TECHNOLOGY (IJERT) Volume 10, Issue 10 (October 2021), 